\title{
CONVENTIONAL RADIOGRAPHY AND MRI DIAGNOSIS OF BONE TUMORS DEVELOPED AT THE KNEE
}

doi: $10.2478 /$ rojost-2018-0038

I. Codorean ${ }^{1}$ I.B. Codorean ${ }^{2}$

${ }^{1}$ Imagistic Center, MedLife Hyper clinic, Grivița, Bucharest, Romania

${ }^{2}$ Orthopaedics \& Traumatology Department, MedLife Hospital, Bucharest, Romania

Introduction. It is known that bone tumors have a predilection to develop for a certain skeletal bone segment. Also, bone tumors have a predilection for certain age groups.

The knee is a common site for bone tumors.

Purpose. Presentation of diagnostic parameters of conventional radiography as the first technique in the detection and characterization of bone tumors developed at the knee level and the criteria for differentiation of the malignant substrate from the benign (the type of bone destruction, the type of periosteal response, poorly defined margins) one. The value of MRI is also presented and illustrated as a unique imaging technique that allows the direct visualization of bone marrow with high spatial resolution for local staging of bone tumors.

Material and method. The present study aimed to investigate the radiographic and MRI imaging characteristics of bone tumors developed at the knee joint, which were retrospectively analyzed (October 2007 and November 2017) in a selected group of 91 patients. The limit of age between 11 and 67 years, $73(70 \%)$ men, $18(30 \%)$ women. Examination protocol: complete clinical examination, radiographic knee examination in anteroposterior and lateral incidents. MRI standard protocol, native, and post paramagnetic contrast.

Results. Conventional Radiographic and MRI have been detected and characterized a number of $55(67 \%)$ primary malignant bone tumors confirmed by histopathological diagnosis, ranging from 8 histopathological types and 37 (33\%) cases of benign tumors with 9 histopathological types. Conclusions. Radiological examination is the first investigation in the evaluation of knee bone tumors, suggesting the malignant nature expressed by the badly defined margin, bone destruction, discontinuous peristaltic reaction, and extension to the soft parts. MRI is a unique imaging technique that allows direct visualization of bone marrow with high spatial resolution and best tool for local staging of bone tumors.

Keywords: knee, bone tumors, conventional radiography, magnetic resonance imaging 